\title{
Genome-wide DNA polymorphisms in Kavuni, a traditional rice cultivar with nutritional and therapeutic properties
}

\begin{tabular}{|r|l|}
\hline Journal: & Genome \\
\hline Manuscript ID & gen-2016-0025.R1 \\
\hline Manuscript Type: & Note \\
\hline Date Submitted by the Author: & 12-Mar-2016 \\
\hline Complete List of Authors: & $\begin{array}{l}\text { Pasupathi, Rathinasabapathi; SRM University, Department of Genetic } \\
\text { Engineering } \\
\text { Natarajan, Purushothaman; SRM University, Department of Genetic } \\
\text { Engineering } \\
\text { Madasamy, Parani; SRM University, Genetic Engineering }\end{array}$ \\
\hline Keyword: & Kavuni, SNPs, medicinal rice, whole genome sequencing \\
\hline & \multicolumn{2}{|c}{} \\
\hline
\end{tabular}


Genome-wide DNA polymorphisms in Kavuni, a traditional rice cultivar with nutritional and therapeutic properties

Pasupathi Rathinasabapathi ${ }^{\#}$, Natarajan Purushothaman ${ }^{\#}$, Madasamy Parani*

Genomics Laboratory, Department of Genetic Engineering, SRM University, Chennai, Tamil

Nadu- 603 203, India.

\# These authors contributed equally to this work

*Corresponding author: Madasamy Parani Email: parani.m@ktr.srmuniv.ac.in 


\begin{abstract}
Although rice genome was sequenced in the year 2002, efforts in resequencing the large number of available accessions, landraces, traditional cultivars, and improved varieties of this important food crop are limited. We have initiated resequencing of the traditional cultivars from India. Kavuni is an important traditional rice cultivar from South India that attracts premium price for its nutritional and therapeutic properties. Whole genome sequencing of Kavuni using Illumina platform and SNPs analysis using Nipponbare reference genome identified 1,150,711 SNPs of which 377,381 SNPs were located in the genic regions. Nonsynonymous SNPs $(62,708)$ were distributed in 19,251 genes, and their number varied between 1 and 115 per gene. Large-effect DNA polymorphisms $(7,769)$ were present in 3,475 genes. Pathway mapping of these polymorphisms revealed the involvement of genes related to carbohydrate metabolism, translation, protein-folding and cell death. Analysis of the starch biosynthesis related genes revealed that the granule-bound starch synthase I gene had T/G SNPs at the first intron/exon junction and a two-nucleotide combination, which were reported to favour high amylose content and low glycemic index. The present study provided a valuable genomics resource to study the rice varieties with nutritional and medicinal properties.
\end{abstract}

Keyword: Kavuni, SNPs, medicinal rice, whole genome sequencing 


\section{Introduction}

Rice is a major staple food for half of the world's population providing up to $75 \%$ of the daily dietary energy intake (Khush, 2005). Besides commercial high yielding rice varieties, a large number of naturally occurring landraces and traditional varieties are cultivated in several parts of the world. Kavuni is an important traditional rice cultivar from South India, which is cultivated for its nutritional and therapeutic properties. The grains of Kavuni are black in colour, and rich in dietary fibre, carotenoids, minerals, and anti-oxidants. Grains of Kavuni contain $95 \%$ higher lutein compared to the improved rice varieties such as IR 64, CO 50 and White Ponni (Valarmathi et al. 2014). Lutein is predominantly found in the macular region of the retina, and consumption of lutein-rich food greatly reduces the risk of developing agerelated macular degeneration (AMD) that leads to blindness (SanGiovanni and Emily, 2007). Methanol extract of Kavuni grains possess alpha-amylase and alpha-glucosidase inhibitory compounds to be useful in the management of type 2 diabetes (Valarmathi et al. 2014). These nutritional and therapeutic properties of Kavuni attracts premium price for farmers. However, the photosensitive nature and the low yield potential of Kavuni limits its large scale cultivation. Therefore, efforts are being made to understand the genetic basis of the desirable traits of Kavuni for introgression into improved verities through marker assisted breeding. Here we report, for the first time, a genome-wide DNA polymorphism study in Kavuni using Illumina's pair-end sequencing. Detailed analysis of the starch biosynthesis related genes (SSRGs) revealed the presence of SNPs that are related to high amylose content and low glycemic index.

\section{Materials and Methods}

Seeds of Oryza sativa L. cv. Kavuni were collected from Kulithalai, Karur district, Tamil Nadu, India. The plants were grown in greenhouse and genomic DNA was extracted from the 
young leaves of a single plant using cetyl trimethyl ammonium bromide (CTAB) method (Murray \& Thompson, 1980). A paired-end genomic library was prepared according to the manufacturer's protocol (Illumina Inc., USA). Adapters were ligated to the ends of the DNA, and DNA clusters were prepared using cBOT and TruSeq PE Cluster kit v3-cBot-HS (Illumina Inc., USA). The DNA clusters were sequenced using Illumina's paired-end sequencing technology on Hiseq 2500 systems (Illumina Inc., USA) to generate $101 \mathrm{bp}$ paired-end reads. The reads were extracted as paired-end reads in FASTQ format for further downstream analysis. The adapters in the paired-end reads were trimmed using 'cutadapt' (https://code.google.com/p/cutadapt/). The trimmed reads were filtered using sickle master (https://github.com/najoshi/sickle) by retaining the bases with minimum Phred quality score of 30. The filtered paired-end reads were mapped to the Oryza sativa L. cv. Nipponbare reference genome (Os-Nipponbare-Reference-IRGSP-1.0) (Kawahara et al. 2013) using Burrows Wheeler alignment (BWA) tool with default parameters (Li and Durbin, 2009). The aligned reads in the SAM file were sorted using SortSam of Picard tool V1.118, and the sorted SAM file was converted to BAM file for variant calling using SAMtools (v0.1.19). The 'mpileup' of SAMtools was used for the identification of SNPs and InDel (Li et al. 2009). The identified DNA polymorphisms were filtered using the following stringent criteria, (1) number of reads per base between 5 and 75, (2) base quality $\geq 30$, (3) mapping quality $\geq 60$, (4) variant quality $\geq 90$, and (5) distance of adjacent variant $\geq 5$. Genomic distribution of DNA polymorphisms was determined by calculating their frequency in $100 \mathrm{~kb}$ interval on each chromosome. The chromosome regions were defined as SNP rich regions if $\mathrm{SNPs} / \mathrm{kb}>5$, and SNP poor regions if SNPs/kb $<0.1$. SnpEff V3.6 tool (Cingolani et al. 2012) was used for annotation of the filtered DNA polymorphisms with rice7 gene model database (http://sourceforge.net/projects/_ snpeff/files/databases/v3_6/ snpEff_v3_6_rice7.zip). Genes harbouring DNA polymorphisms that affected the translation 
of the encoded proteins were identified in the Kyoto Encyclopaedia of Genes and Genomes database (KEGG; http://www.genome.jp/kegg) and mapped to pathways. Further, gene ontology enrichment analysis was performed using BiNGO tool with p-value cut-off of $\leq 0.05$ (Maere et al. 2005). The unmapped reads were extracted into a BAM file using SAMtools and the reads in the BAM file were converted to FASTQ format using BAMtools V2.3.0. The unmapped FASTQ reads were assembled into contigs by de novo using Velvet V1.2.10 (Zerbino and Birney, 2008). The assembly was optimized using different Kmer sizes, and all the contigs were used for further analysis. The de novo assembled contigs were subjected to $\begin{array}{lllll}\text { Gene } & \text { Ontology } & \text { (GO) } & \text { analysis } & \text { using }\end{array}$ (https://www.arabidopsis.org/tools/bulk/go/index.jsp).

\section{Results and Discussion}

Whole genome sequencing of Kavuni rice variety yielded $42 \times 10^{6}$ paired-end reads, which were reference assembled to Oryza sativa L. cv. Nipponbare reference genome (IRGSP1.0.) (Fig. S1). In total, $91.0 \%$ of the reads were mapped to the reference genome, which included $77.7 \%$ uniquely mapped reads that covered $83.3 \%$ of the genome (Table S1). The BAM files generated for this study were submitted to the sequence read archive (SRA) at NCBI with the accession number PRJNA296443. Analysis of the uniquely mapped reads under stringent filtering conditions showed the presence of 1,150,711 SNPs and 104,984 InDels. The average number of DNA polymorphisms per chromosome was 104,641 with chromosomes 1 and 9 having the highest $(151,294)$ and lowest $(77,029)$ number of polymorphisms, respectively. The frequency of DNA polymorphisms were determined for every $100 \mathrm{~kb}$ of the genome (Fig. S2), and 344 SNP rich regions and 164 SNP poor regions were identified (Table S2). All the chromosomes were found to have SNP rich and SNP poor regions, and the longest SNP rich region was identified on chromosome 11 between 18.2 and $18.5 \mathrm{Mb}(0.4$ 
$\mathrm{Mb}$ ). The longest SNP poor region was identified on chromosome 5 between 9.6 and $13.0 \mathrm{Mb}$ $(3.4 \mathrm{Mb})$ in a similar region reported in other rice varieties (Hu et al. 2014; Jain et al. 2014; Wang et al. 2009). Such contiguous regions with abnormally low SNPs may represent adaptive gene selection during domestication and crop improvement of rice (Krishnan et al. 2014). A higher frequency of transition over transversion SNPs were observed in Kavuni with an average transition to transversion SNP ratio of 2.2. Such transition bias was previously reported in rice, and the ratio varied between 2.0 and 2.53 (Mace et al. 2013; Xu et al. 2012).

Annotation of DNA polymorphisms revealed the presence of 377,381 SNPs in the genic regions of the Kavuni genome (Table 1). Coding regions contained 107,938 SNPs, which included 62,708 non-synonymous SNPs that were distributed in 19,251 genes. The number of non-synonymous SNPs per gene varied between 1 and 115. The ratio between nonsynonymous to synonymous SNP in the Kavuni genome was 1.39 , which was reported to vary between 1.14 and 1.39 in other rice varieties (Arai-Kichise et al. 2011; Jeong et al. 2013; Rathinasabapathi et al. 2015; Subbaiyan et al. 2012). The Kavuni genome contained 2,451 large effect SNPs (loss of start codon, introduction of premature stop codon, and loss of stop codon) and 5,318 frame shift mutations in 3,475 genes that may affect the protein functions. KEGG pathway mapping identified 317 genes that were related to different biological functions such as signal transduction pathway, carbohydrate metabolism, protein folding and cell death (Fig. S3). Gene ontology (GO) enrichment analysis showed that the genes were enriched for the biological processes such as oligo-peptide transport, amino acid phosphorylation and localization. At the molecular function level, the genes for serine/threonine kinase activity, ATP binding, and metallo-endopeptidase activity were found to be enriched (Fig. S4). 
High amylose content in rice grains result in low glycemic index (GI), which is desirable for the management of postprandial hyperglycemia in diabetes patients (Fitzgerald et al. 2011; Silva, et al. 2015). Therefore, starch biosynthesis pathway that favours amylose over amylopectin accumulation is a desirable health benefit. Starch biosynthesis in rice involves seven enzymes encoded by 18 starch biosynthesis related genes (SSRGs) (Tian et al. 2009). The SSRGs in the Kavuni genome contained 355 SNPs including 29 non-synonymous SNPs and 45 InDels (Table 2). The granule-bound starch synthase I gene (GBSSI) in particular contained $\mathrm{T} / \mathrm{G}$ non-coding SNPs at the intron/exon 1 junction site, and a two-nucleotide combination (' $\mathrm{A}$ ' at position 2,386 and ' $\mathrm{C}$ ' at position 3,378), which were reported to be associated with highest amylose content in rice varieties and breeding populations (Kharabian Masouleh, 2013; Kharabian-Masouleh et al. 2011; Larkin and Park, 1999; Rathinasabapathi et al. 2015). A significant portion of the indica genome does not map to the japonica reference genome due to the natural genetic divergence between the two sub-species (Jain et al. 2014; Subbaiyan et al. 2012; Tang et al. 2006). Therefore, the unmapped reads of Kavuni (9.07\%) were de novo assembled into contigs. The results showed 797 contigs that ranged in size between 120 and 6,188 bp. These contigs were subjected to annotation and Gene Ontology (GO) analysis in terms of cellular, biological and molecular functions. More number of contigs were related to transferase activity, macromolecular binding, and kinase activity (molecular processes) or cellular and metabolic processes (biological processes) or nucleus, cytoplasmic, intracellular and membrane components (cellular components) (Fig. S5).

Acknowledgement: This study was financially supported by SRM-DBT Partnership Platform for Contemporary Research Services and Skill Development in Advanced Life Sciences Technologies (Order No. BT/PR12987/INF/22/205/2015). We also acknowledge the 
SciGenom, Cochin, India for their technical support in whole genome sequencing.

\section{Reference}

Arai-Kichise, Y., Shiwa, Y., Nagasaki, H., Ebana, K., Yoshikawa, H., Yano, M., and Wakasa, K. 2011. Discovery of genome-wide DNA polymorphisms in a landrace cultivar of Japonica rice by whole-genome sequencing. Plant Cell Physiol. 52(2): 274-82. doi: $10.1093 / \mathrm{pcp} / \mathrm{pcr} 003$

Cingolani, P., Platts, A., Wang, L. L., Coon, M., Nguyen, T., Wang, L., Coon, M., Nguyen, T., Wang, L., Land, S. J., Ruden, D.M., and Lu, X. 2012. A program for annotating and predicting the effects of single nucleotide polymorphisms, SnpEff: SNPs in the genome of Drosophila melanogaster strain w1118; iso-2; iso-3. Fly, 6(2): 1-13. doi: 10.4161/fly.19695.

Fitzgerald, M. A., Rahman, S., Resurreccion, A. P., Concepcion, J., Daygon, V. D., Dipti, S. S., Kabir, K.A., Klingner, B., Morell, M.K., and Bird, A. R. 2011. Identification of a Major Genetic Determinant of Glycaemic Index in Rice. Rice. 4(2): 66-74. doi: 10.1007/s12284-011-9073-Z

Hu, Y., Mao, B., Peng, Y., Sun, Y., Pan, Y., Xia, Y., Sheng, X., Yuan, L., and Zhao, B. 2014. Deep re-sequencing of a widely used maintainer line of hybrid rice for discovery of DNA polymorphisms and evaluation of genetic diversity. Mol. Genet. Genomics. doi: 10.1007/s00438-013-0807-z.

Jain, M., Moharana, K. C., Shankar, R., Kumari, R., and Garg, R. 2014. Genomewide discovery of DNA polymorphisms in rice cultivars with contrasting drought and salinity stress response and their functional relevance. Plant Biotech. J. 12(2): 253-64. doi: 10.1111/pbi.12133.

Jeong, I.S., Yoon, U.H., Lee, G.S., Ji, H.S., Lee, H.J., Han, C.D., Hahn, J., An, G., and Kim, 
T.H. 2013. SNP-based analysis of genetic diversity in anther-derived rice by whole genome sequencing. Rice. 6(1): 6. doi: 10.1186/1939-8433-6-6.

Kawahara, Y., Bastide, M. De, Hamilton, J. P., Kanamori, H., Mccombie, W. R., Ouyang, S., Schwartz, D.C., Tanaka, T., Wu, J., Zhou, S., Child, K.L., Davidson, R.M., Lin, H., Quesada-Ocampo, L., Vaillancourt, B., Sakai, H., Lee, S.S., Kim, J., Numa, H., Itoh, T., Buell, C.R., and Matsumoto, T. 2013. Improvement of the Oryza sativa Nipponbare reference genome using next generation sequence and optical map data. Rice, 6(4): 110. doi:10.1186/1939-8433-6-4.

Kharabian Masouleh, A. 2013. Characterisation of starch traits and genes in Australian rice germplasm. Ph.D thesis, South Cross Plant Science, South Cross University, New South Wales, Australia.

Kharabian-Masouleh, A., Waters, D. L. E., Reinke, R. F., and Henry, R. J. (2011). Discovery of polymorphisms in starch-related genes in rice germplasm by amplification of pooled DNA and deeply parallel sequencing. Plant Biotech. J. 9(9): 1074-85. doi: 10.1111/j.1467-7652.2011.00629.x

Khush, G. S. 2005. What it will take to Feed 5.0 Billion Rice consumers in 2030. Plant Mol. Bio. 5(9): 1-6. doi: 10.1007/s11103-005-2159-5.

Krishnan S, G., Waters, D. L. E., and Henry, R. J. 2014. Australian wild rice reveals predomestication origin of polymorphism deserts in rice genome. PloS One. 9(6): e98843. doi: 10.1371/journal.pone.0098843.

Larkin, P. D., and Park, W. D. 1999. Transcript accumulation and utilization of alternate and non-consensus splice sites in rice granule-bound starch synthase are temperaturesensitive and controlled by a single-nucleotide polymorphism. Plant Mol. Bio. 40(4): 719-27. Available from from http://www.ncbi.nlm.nih.gov/pubmed/10480395.

Li, H., and Durbin, R. 2009. Fast and accurate short read alignment with Burrows-Wheeler 
transform. Bioinformatics. 25(14): 1754-1760. doi: 10.1093/bioinformatics/btp324.

Heng Li1,†, Bob Handsaker2,†, Alec Wysoker2, Tim Fennell2, Jue Ruan3, Nils Homer4, Gabor Marth5, Goncalo Abecasis6, Richard Durbin1

Li, H., Handsaker, B., Wysoker, A., Fennell, T., Ruan, J., Homer, N., Marth, G., Abecasis, G., and Durbin, R. 2009. The Sequence Alignment/Map format and SAMtools. Bioinformatics, 25(16): 2078-2079. doi: 10.1093/bioinformatics/btp352.

Mace, E. S., Tai, S., Gilding, E. K., Li, Y., Prentis, P. J., Bian, L., Campbell, B.V., Hu, W., Innes, D.J., Han, X., Cruickshank, A., Dai, C., Frere, C., Zhang, H, Hunt, C.H., Wang, X., Shatte, T., Wang, M., Su, Zhe., Li, J., Lin, X., Godwin, I.D., Jordan, D.R., and Wang, J. 2013. Whole-genome sequencing reveals untapped genetic potential in Africa's indigenous cereal crop sorghum. Nature Commun. 4(2320): 1-9. doi: $10.1038 /$ ncomms 3320

Maere, S., Heymans, K., and Kuiper, M. 2005. BiNGO: A Cytoscape plugin to assess overrepresentation of Gene Ontology categories in Biological Networks. Bioinformatics. 21(16): 3448-3449. doi: 10.1093/bioinformatics/bti551.

Murray, M. G., and Thompson, W. F. 1980. Rapid isolation of high molecular weight plant DNA. Nucleic Acids Res. 8(19): 4321-4325. Available from http://www. ncbi.nlm. nih. gov/pubmed/7433111.

Rathinasabapathi, P., Purushothaman, N., Ramprasad, V., and Parani, M. 2015. Whole genome sequencing and analysis of Swarna, a widely cultivated indica rice variety with low glycemic index. Sci. Rep. 5( 11303): 1-10. doi: 10.1038/srep11303.

SanGiovanni, S., and Emily, Y. 2007. The relationship of dietary carotenoid and vitamin A, $\mathrm{E}$, and $\mathrm{C}$ intake with age-related macular degeneration in a case-control study. Arch. Ophthalmol. 125(9):1225-1232. doi:10.1001/archopht.125.9.1225.

Silva, F. M., Kramer, C. K., Crispim, D., and Azevedo, M. J. 2015. A High-Glycemic Index, 
Low-Fiber Breakfast Affects the Postprandial Plasma Glucose, Insulin, and Ghrelin Responses of Patients with Type 2 Diabetes in a Randomized Clinical Trial. J. Nutr. 110. doi: 10.3945/jn.114.195339.

Subbaiyan, G. K., Waters, D. L. E., Katiyar, S. K., Sadananda, A. R., Vaddadi, S., and Henry, R. J. 2012. Genome-wide DNA polymorphisms in elite indica rice inbreds discovered by whole-genome sequencing. Plant Biotech.J. 10(6), 623-34. doi: 10.1111/j.14677652.2011.00676.x.

Tang, T., Lu, J., Huang, J., He, J., McCouch, S. R., Shen, Y., Kai, Z., Purugganan, M.D., Shi, S., and $\mathrm{Wu}, \mathrm{C}$. I. 2006. Genomic variation in rice: Genesis of highly polymorphic linkage blocks during domestication. PLoS Genetics. 2(11); 1824-1833. doi: 10.1371/journal.pgen.0020199.

Tian, Z., Qian, Q., Liu, Q., Yan, M., Liu, X., Yan, C., Liu, X., Yan, C., Liu, G., Gao, Z., Tang, S., Zeng, D., Wang, Y., Yu, J., Gu, M., and Li, J. 2009. Allelic diversities in rice starch biosynthesis lead to a diverse array of rice eating and cooking qualities. Proc. Natl. Acad. Sci. USA. 106(51): 21760-21765. doi: 10.1073?pnas.0912396106.

Valarmathi, Raveendran, R., Robin, S., and Senthil, N. 2014. Unraveling the nutritional and therapeutic properties of "Kavuni" a traditional rice variety of Tamil Nadu. J.Plant Biochem. Biotech. 24(3): 305 -315. doi: 10.1007/s13562-014-0274-6.

Wang, L., Hao, L., Li, X., Hu, S., Ge, S., and Yu, J. 2009. SNP deserts of Asian cultivated rice: Genomic regions under domestication. J. Evol. Bio. 22(4): 751-761. doi: 10.1111/j.1420-9101.2009.01698.x.

Xu, X., Liu, X., Ge, S., Jensen, J. D., Hu, F., Li, X., Dong, Y., Gutenkunst, R.N., Fang, L., Huang, L., Li, J., He, W., Zhang, G., Zheng, X., Zhang, F., Li, Y., Yu, C., Kristiansen, K., Zhang, X., Wang, J., Wright, M., McCouch, S., Nielsen, R., Wang, J., and Wang, W. 2012. Resequencing 50 accessions of cultivated and wild rice yields markers for 
identifying agronomically important genes. Nature Biotech. 30(1): 105-11. doi:10.1038/nbt.2050.

Zerbino, D. R., and Birney, E. 2008. Velvet: Algorithms for de novo short read assembly using de Bruijn graphs. Genome Res. 18(5): 821-829. doi: 10.1101/gr.074492.107. 
Table 1: Annotation of SNPs and InDels that were identified in Kavuni.

\begin{tabular}{lll}
\hline & SNPs & InDels \\
\hline Total & $1,150,711$ & 104,984 \\
Intergenic & 773,330 & 61,690 \\
Genic & 377,381 & 49,294 \\
Intron and regulatory sequences & 223,886 & 21,483 \\
UTRs & 45,557 & 18,229 \\
Coding region & 107,938 & 3,582 \\
$\quad$ Synonymous SNPs & 45,230 & \\
\multicolumn{2}{l}{ Non-synonymous SNPs } & 62,708 \\
\hline
\end{tabular}


Table 2: Details of the SNPs and InDels that were identified in the starch biosynthesis related genes (SSRG) of Kavuni.

\begin{tabular}{|c|c|c|c|c|c|c|}
\hline S.No & Gene Name & $\begin{array}{l}\text { Non- } \\
\text { Coding } \\
\text { SNPs }\end{array}$ & $\begin{array}{c}\text { Non- } \\
\text { Synon } \\
\text { ymous } \\
\text { SNPs } \\
\end{array}$ & $\begin{array}{c}\text { Synon } \\
\text { ymous } \\
\text { SNPs }\end{array}$ & $\begin{array}{c}\text { Non- } \\
\text { coding } \\
\text { InDels }\end{array}$ & $\begin{array}{l}\text { Coding } \\
\text { InDels }\end{array}$ \\
\hline 1 & $\begin{array}{l}\text { ADP- glucose pyrophosphorylase } \\
\text { (small unit) }\end{array}$ & 16 & 0 & 3 & 1 & 0 \\
\hline 2 & Alpha 1,4- glucanphosphorylase & 0 & 0 & 0 & 1 & 0 \\
\hline 3 & Glucose 6-phosphate-translocator & 10 & 1 & 3 & 3 & 0 \\
\hline 4 & Granule-bound starch synthase I & 1 & 0 & 1 & 1 & 0 \\
\hline 5 & Granule-bound starch synthase II & 67 & 11 & 2 & 7 & 0 \\
\hline 6 & Starch synthase I & 60 & 2 & 7 & 5 & 0 \\
\hline 7 & Starch synthase IIa & 17 & 1 & 3 & 1 & 0 \\
\hline 8 & Starch synthase IIb & 8 & 2 & 1 & 3 & 0 \\
\hline 9 & Starch synthase IIIa & 19 & 2 & 4 & 3 & 0 \\
\hline 10 & Starch synthase IIIb & 8 & 4 & 1 & 1 & 0 \\
\hline 11 & Starch synthase IVa & 9 & 2 & 1 & 1 & 0 \\
\hline 12 & Starch synthase IVb & 17 & 0 & 4 & 1 & 0 \\
\hline 13 & Branching enzyme I & 9 & 0 & 1 & 2 & 0 \\
\hline 14 & Branching enzyme IIa & 0 & 0 & 0 & 0 & 0 \\
\hline 15 & Branching enzyme IIb & 24 & 2 & 3 & 3 & 0 \\
\hline 16 & Debranching enzyme -isoamylase 1 & 11 & 1 & 3 & 1 & 0 \\
\hline 17 & Debranching enzyme -isoamylase 2 & 1 & 1 & 1 & 0 & 0 \\
\hline \multirow[t]{2}{*}{18} & Debranching enzyme -Pullulanase & 214 & 0 & 22 & 11 & 0 \\
\hline & Total/ average & 266 & 29 & 60 & 45 & 0 \\
\hline
\end{tabular}


Genome-wide DNA polymorphisms in Kavuni, a traditional rice cultivar with nutritional and therapeutic properties

\section{Pasupathi Rathinasabapathi ${ }^{\#}$, Natarajan Purushothaman ${ }^{\#}$, Madasamy Parani*}

Genomics Laboratory, Department of Genetic Engineering, SRM University, Chennai, Tamil

Nadu- 603 203, India.

\# These authors contributed equally to this work

*Corresponding author: Madasamy Parani Email: parani.m@ktr.srmuniv.ac.in 


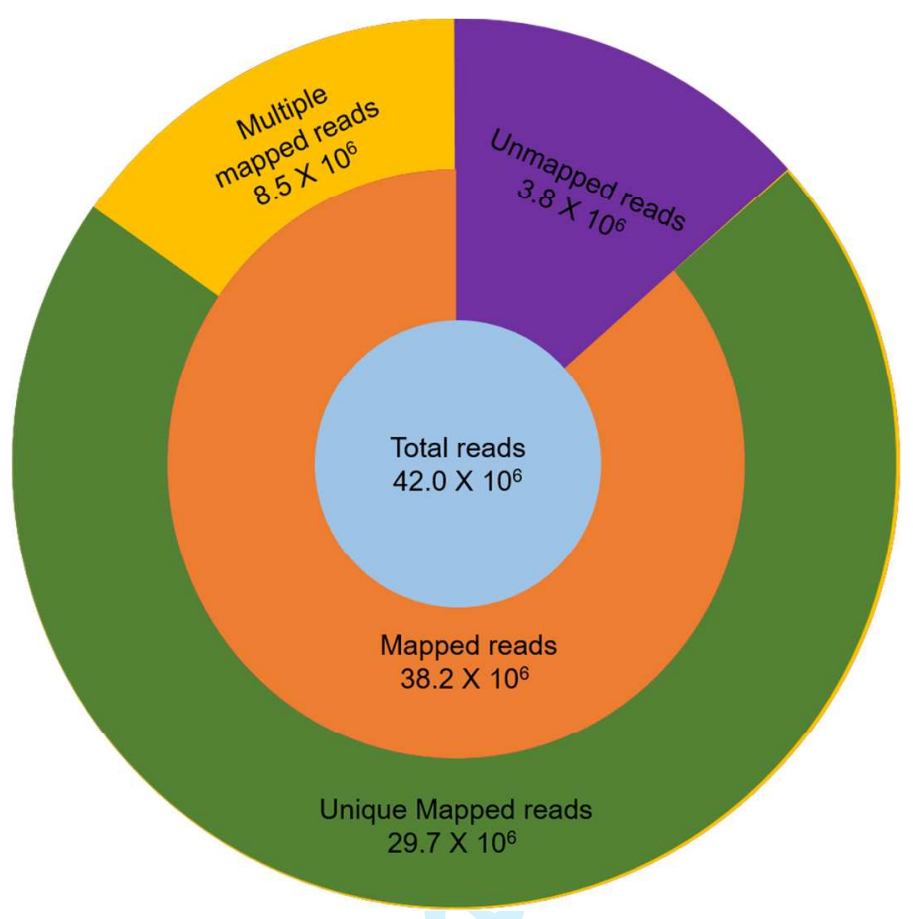

Fig. S1. Classification of the Kavuni reads mapped on to the Nipponbare reference genome. The total number reads in the centre circle. Number of reads mapped on the nuclear genome and unmapped reads in the middle circle. The outer circle represents the uniquely and multiple mapped reads. 


$$
\begin{aligned}
& \text { Ch8.01 }
\end{aligned}
$$

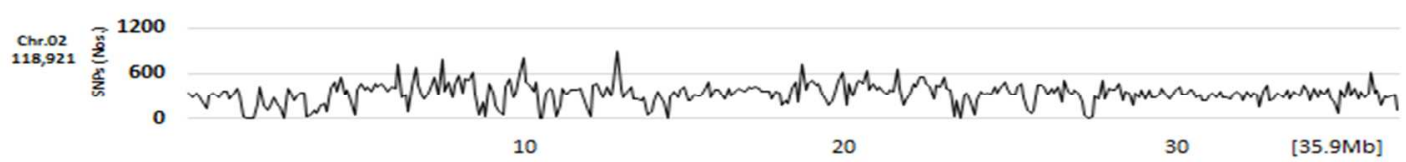

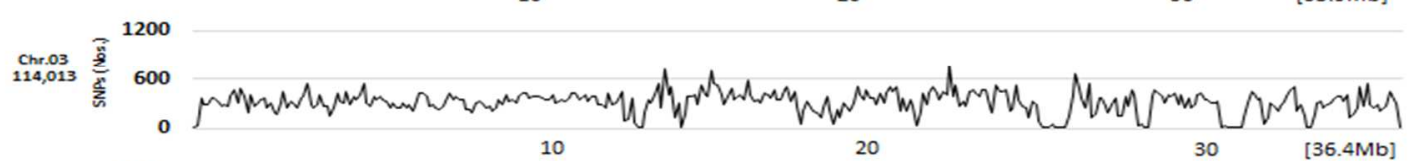

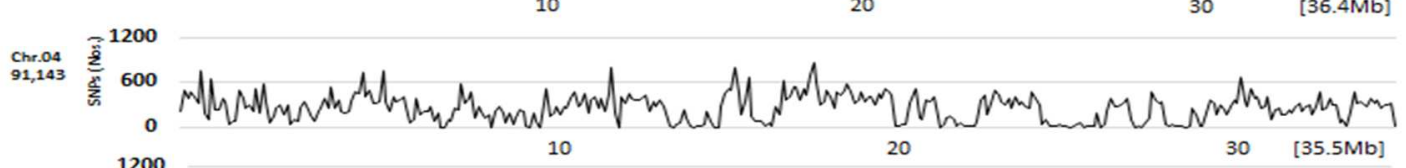

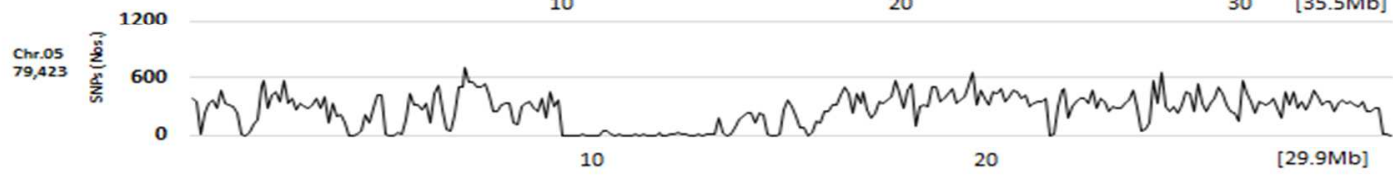

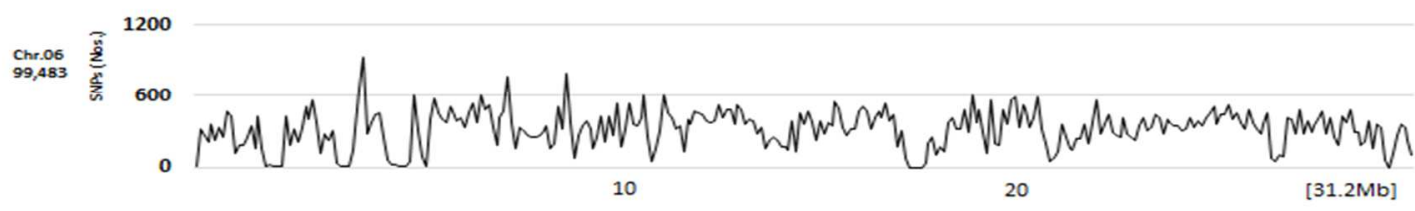

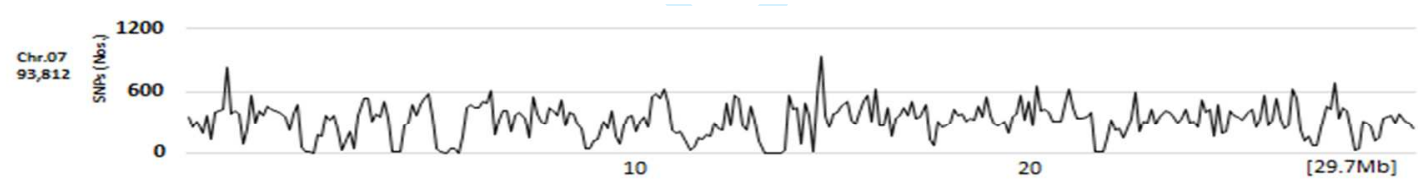

$$
\begin{aligned}
& \text { (28.4Mb] }
\end{aligned}
$$

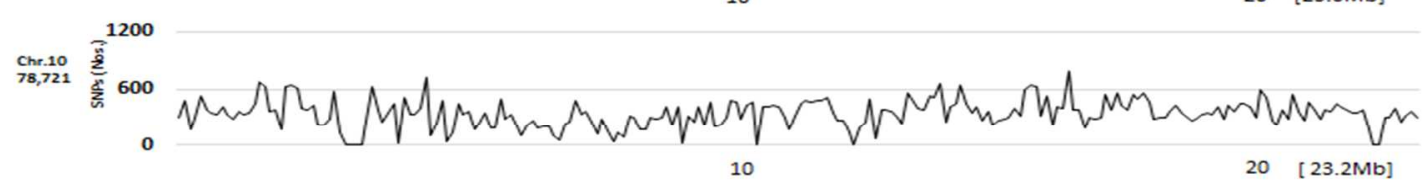

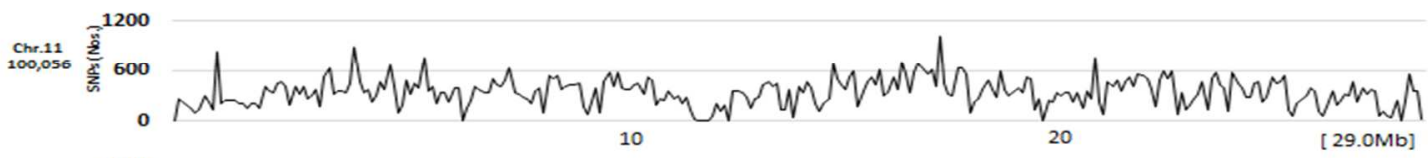

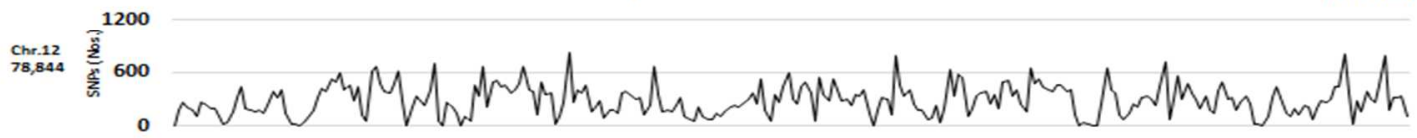

Fig. S2. Frequency of SNPs detected in Kavuni in each chromosome. The x-axis represented the physical distance along each chromosome, split into $100 \mathrm{~kb}$ windows. The total size of each chromosome was shown in brackets. The y-axis indicated the number of SNPs. The total number of SNPs in each chromosome was mentioned. 


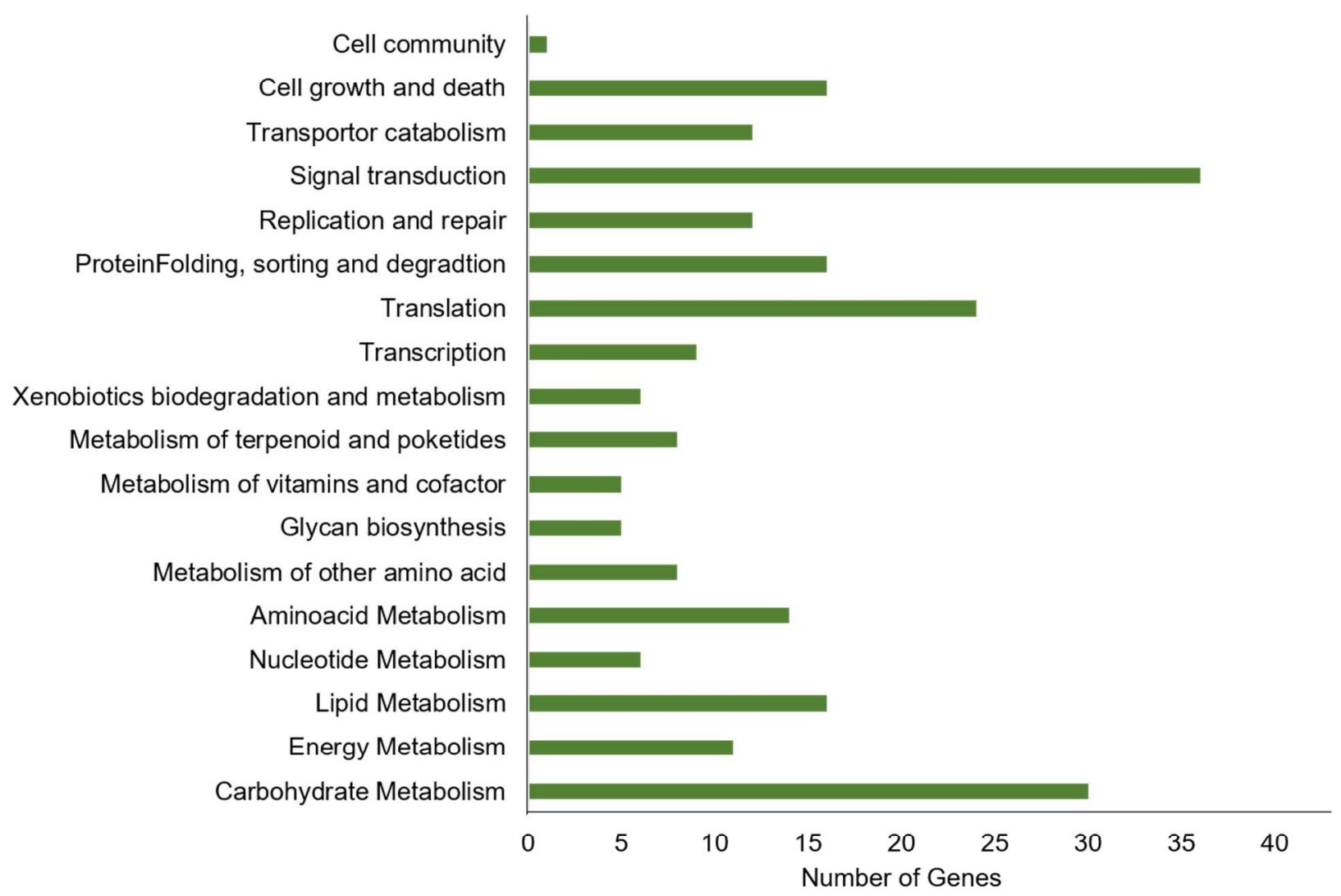

Fig. S3. Pathway mapping of large effect DNA polymorphism in rice using Kyoto Encyclopedia of Genes and Genomes (KEGG) database. 
A)

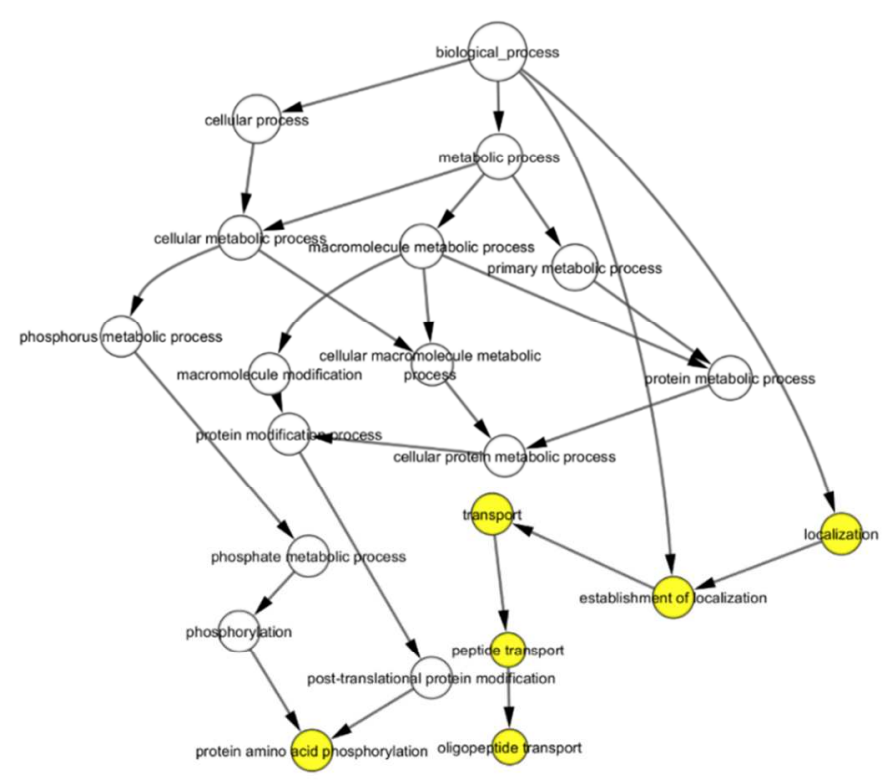

B)

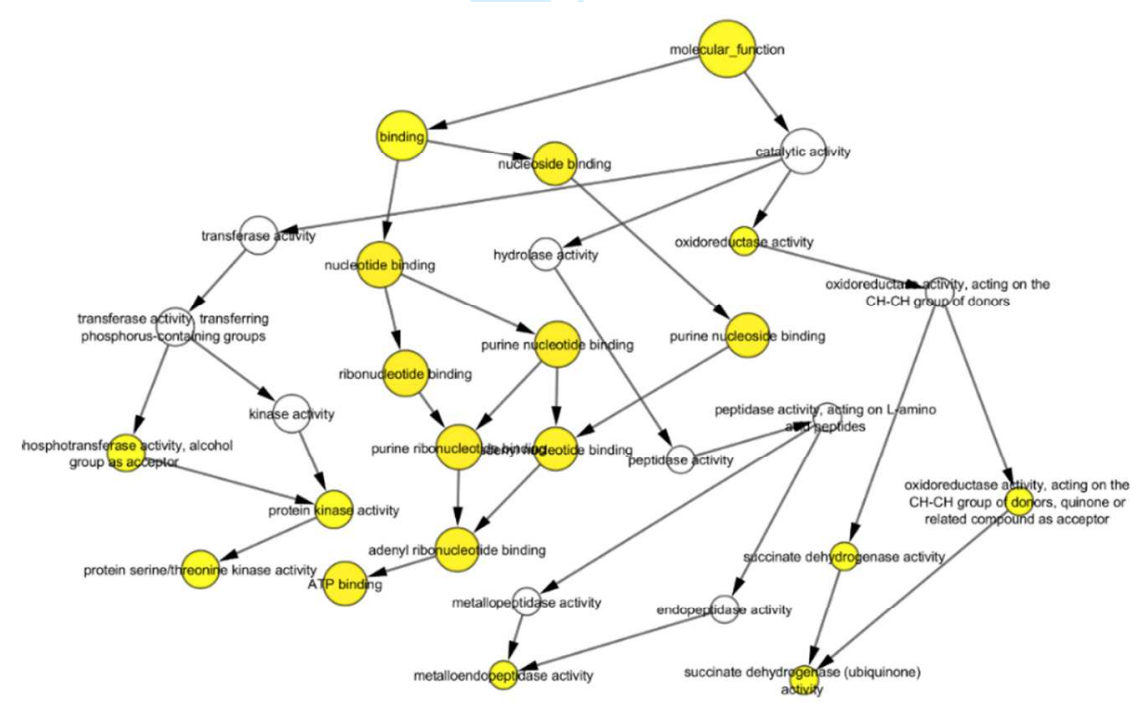

Fig. S4. Analysis of gene ontology term enrichment of the genes detected with large-effect DNA polymorphisms. Node size is proportional to the number of transcripts in each category and colours shaded according to the significance level (white - no significant difference; Yellow scale - significantly enriched). A) Biological process B) Molecular function. 


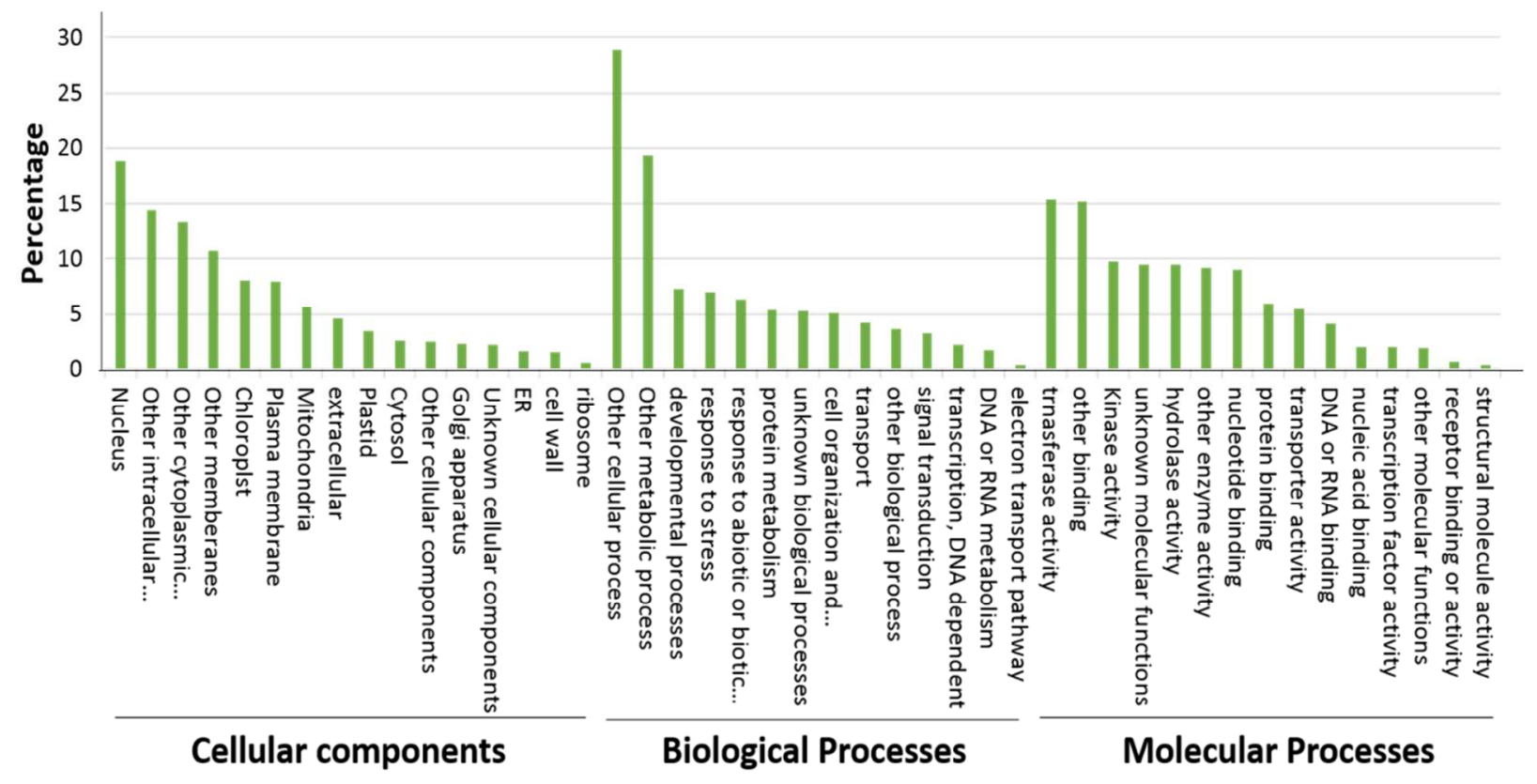

Fig. S5. Functional categorization of the contigs assembled from the unmapped reads of Kavuni into gene ontology (GO) terms: biological processes, cellular components, molecular functions. 
Table S1: Mapping and coverage of the reads from Kavuni to the Nipponbare reference genome IRGSP1.0

\begin{tabular}{|c|c|c|c|c|}
\hline Chromosome & $\begin{array}{l}\text { Nipponbare } \\
\text { genome (bp) }\end{array}$ & $\begin{array}{c}\text { Uniquely } \\
\text { Mapped Reads }\end{array}$ & $\begin{array}{c}\text { Uniquely covered } \\
\text { bases }\end{array}$ & Coverage (\%) \\
\hline 1 & 43270923 & 3440333 & 37422819 & 86.5 \\
\hline 2 & 35937250 & 2919632 & 31416258 & 87.4 \\
\hline 3 & 36413819 & 3054972 & 32789741 & 90.0 \\
\hline 4 & 35502694 & 2710415 & 28335645 & 79.8 \\
\hline 5 & 29958434 & 2491118 & 26353056 & 88.0 \\
\hline 6 & 31248787 & 2454017 & 25798844 & 82.6 \\
\hline 7 & 29697621 & 2278588 & 24296820 & 81.8 \\
\hline 8 & 28443022 & 2282285 & 23599317 & 83.0 \\
\hline 9 & 23012720 & 1793538 & 19216723 & 83.5 \\
\hline 10 & 23207287 & 2022948 & 19042193 & 82.1 \\
\hline 11 & 29021106 & 2077217 & 22352387 & 77.0 \\
\hline 12 & 27531856 & 2134555 & 21306714 & 77.4 \\
\hline Total/Avg. & 373245519 & 29659618 & 311930517 & 83.3 \\
\hline
\end{tabular}


Table S2: Number and densities of SNPs and InDels in the genome of Kavuni.

\begin{tabular}{|c|c|c|c|c|}
\hline \multirow{2}{*}{ Chromosome } & \multicolumn{2}{|c|}{ SNPs } & \multicolumn{2}{|c|}{ InDels } \\
\hline & Nos. & Density & Nos. & Density \\
\hline 1 & 138,240 & 318.8 & 13,054 & 29.9 \\
\hline 2 & 118,921 & 329.8 & 11,195 & 30.8 \\
\hline 3 & 114,013 & 312.0 & 11,058 & 30.0 \\
\hline 4 & 91,143 & 255.6 & 7,721 & 21.5 \\
\hline 5 & 79,421 & 265.2 & 7,550 & 25.1 \\
\hline 6 & 99,483 & 317.4 & 9,103 & 28.8 \\
\hline 7 & 93,812 & 315.4 & 8,677 & 28.9 \\
\hline 8 & 87,212 & 305.6 & 8,018 & 27.9 \\
\hline 9 & 70,845 & 307.5 & 6,184 & 26.7 \\
\hline 10 & 78,721 & 338.7 & 6,878 & 29.4 \\
\hline 11 & 100,056 & 343.1 & 8,597 & 29.2 \\
\hline 12 & 78,844 & 285.2 & 6,949 & 24.9 \\
\hline otal/Avg. & $1,150,711$ & 307.9 & 104,984 & 27.7 \\
\hline
\end{tabular}

